\title{
Involvement of cellular metabolism in age-related LTP modifications in rat hippocampal slices
}

\author{
Dominika Drulis-Fajdasz ${ }^{1}$, Tomasz Wójtowicz ${ }^{2}$, Marcin Wawrzyniak ${ }^{3}$, Jakub \\ Wlodarczyk ${ }^{3}$, Jerzy W. Mozrzymas ${ }^{1,2}$ and Dariusz Rakus ${ }^{1}$ \\ ${ }^{1}$ Department of Animal Molecular Physiology, Institute of Experimental Biology, Wroclaw University, Wroclaw, Poland \\ ${ }^{2}$ Laboratory of Neuroscience, Department of Biophysics, Wroclaw Medical University, Wroclaw, Poland \\ ${ }^{3}$ Laboratory of Cell Biophysics, Department of Molecular and Cellular Neurobiology, Nencki Institute of Experimental Biology, \\ Warsaw, Poland \\ Correspondence to: Dariusz Rakus, email: dariusz.rakus@uni.wroc.pl \\ Jerzy W. Mozrzymas, email: jerzy.mozrzymas@umed.wroc.pl
}

Keywords: aging, plasticity, glycogen synthase, glycogen phosphorylase, dendritic spine maturation

Received: March 05, $2015 \quad$ Accepted: May 12, 2015

Published: May 19, 2015

This is an open-access article distributed under the terms of the Creative Commons Attribution License, which permits unrestricted use, distribution, and reproduction in any medium, provided the original author and source are credited.

\section{ABSTRACT}

Recent studies emphasized crucial role of astrocytic glycogen metabolism in regulation of synaptic transmission and plasticity in young animals. However, the interplay between age-related synaptic plasticity impairments and changes in energetic metabolism remains obscure. To address this issue, we investigated, in hippocampal slices of young (one month) and aged rats (20-22-months), the impact of glycogen degradation inhibition on LTP, mRNA expression for glycogen metabolism enzymes and morphology of dendritic spines. We show that, whereas in young hippocampi, inhibition of glycogen phosphorolysis disrupts the late phase of LTP in the Schaffer collateral-CA1 pathway, in aged rats, blockade of glycogen phosphorylase tends to enhance it. Gene expression for key energy metabolism enzymes, such as glycogen synthase and phosphorylase and glutamine synthetase showed marked differences between young and aged groups and changes in expression of these enzymes preceded plasticity phenomena. Interestingly, in the aged group, a prominent expression of these enzymes was found also in neurons. Concluding, we show that LTP in the considered pathway is differentially modulated by metabolic processes in young and aging animals, indicating a novel venue of studies aiming at preventing cognitive decline during aging.

\section{INTRODUCTION}

Impaired synaptic plasticity is believed to be one of the major mechanisms underlying age-dependent cognitive decline [1]. The majority of excitatory transmission in the brain is mediated by glutamate-gated channels and it is widely accepted that decreased number, deficient trafficking and/or functional impairment of these receptors (especially of AMPA type) contribute to age-related plasticity decline [2]. In addition, it is known that synaptic plasticity phenomena are also manifested by profound alterations in spine shape [3, 4] but little is known how these structures change their propensity to morphological changes with age.

During the past decade a growing body of evidence has accumulated that astrocytes regulate properties of neuronal networks by supplying neurotransmitter precursors and by modulating neuronal signaling by e.g. releasing a broad spectrum of gliotransmitters [5]. Just recently it turned out that energetic metabolism of astrocytes is a crucial player in regulation of basal transmission and the plasticity phenomena in neuronal networks: the inhibition of glycogen breakdown and lactate release from astrocytes reduces the miniature excitatory postsynaptic currents in astrocyte-neuron cocultures [6], inhibits memory consolidation in chicks [7] and disrupts memory formation in mice hippocampi [8]. However, it should be born in mind that slice or behavioral experiments $[8,9]$ were carried out on young or juvenile animals. Importantly, aging is known to affect the stability 
of long-term potentiation, LTP [10], a cellular mechanism of learning and memory (for review see in: [2]) and to alter the expression of energy metabolism enzymes in astrocytes [11]. However, the interplay between agerelated synaptic plasticity impairments and changes in astrocyte energetic metabolism remains obscure. To address this issue, we have investigated, in hippocampal slices of young (one month) and aged rats (20-22-month), the impact of pharmacological blockade of glycogen degradation in astrocytes on LTP, mRNA expression for glycogen metabolism enzymes and the density and morphology of dendritic spines. Our results demonstrate that, in contrast to young hippocampi, inhibition of glycogen phosphorolysis elevates LTP magnitude in Schaffer collateral-CA1 (Sch-CA1) pathway in aged rats. The observed differences in the LTP amplitude and maintenance are accompanied by a different mode of dendritic spines maturation in young and aged animals. The results presented here also suggest that molecular changes in astrocytes energy metabolism precede plasticity phenomena, both in young and aged animals.

In conclusion, we show that LTP in the hippocampal Sch-CA1 pathway is differentially modulated by astrocytic metabolism in young and aging animals, indicating a novel venue of studies aiming at preventing memory problems and cognitive decline during aging.

\section{RESULTS}

\section{Glycogen phosphorylase inhibitor oppositely influences LTP induction in young and aged rats}

Since it may be expected that young and aged animals could show major differences both in neuronal excitability in the selected pathway and in the synaptic transmission, these two aspects were investigated. Analysis of the fiber volley amplitude, which represents the number of stimulated axons firing action potential, revealed no significant difference between young and aged rats for the whole range of stimulating currents $(0$ - $300 \mu \mathrm{A} ; F_{(2,335)}=0.462, p=0.95 ; n=$ at least 10 slices, Figure 1A). To describe basal synaptic function in young and aged animals, we measured the I-O relationships for fEPSPs recorded in the stratum radiatum of CA1
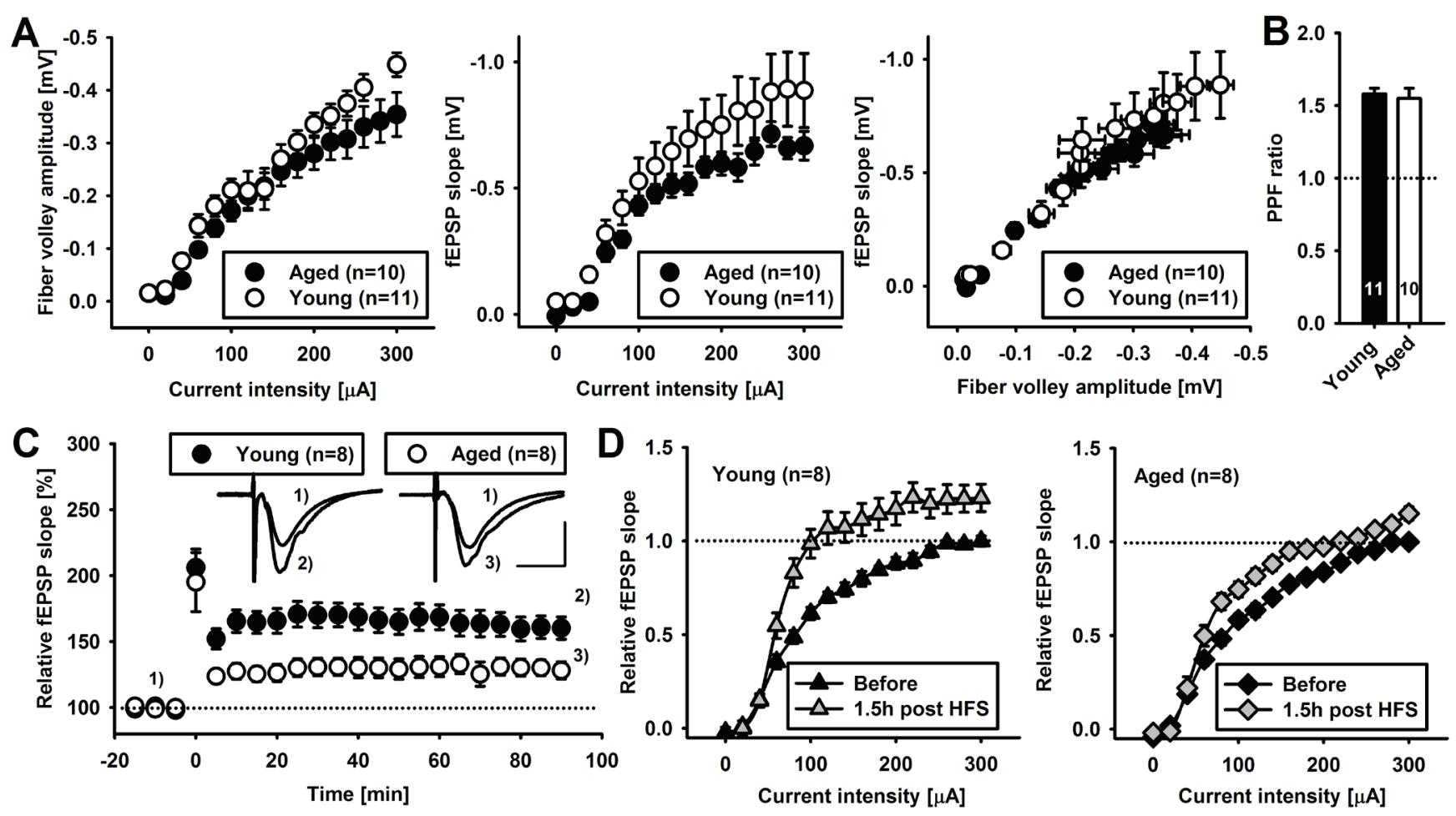

Figure 1: Acute hippocampal brain slices retain key properties during ageing. A. Relationships between the mean fiber volley amplitude, fEPSP slope and stimulating current intensity in young (black circles) and aged (white circles) rats. Note that ageing does not significantly change basal properties of Sch-CA1 excitatory transmission. B. Average basal paired pulse facilitation ratio (50 ms gap) of fEPSP slope in young and aged animals. C. Average time-course of fEPSP slopes recorded in young (black circles) and aged animals (white circles) before and after tetanization $(4 \times 100 \mathrm{~Hz}$, time 0 minutes) normalized to baseline values. Insets show exemplary fEPSPs recorded before (1) and 90 minutes $(2,3)$ after tetanization (scale: $0.4 \mathrm{mV}, 10 \mathrm{~ms}$ ). Note an increased LTP magnitude in young compared to aged animals. D. Average fEPSP slopes plotted against current stimulus (input-output curve, I-O) before (black) and 90 minutes post tetanization (grey) in young (left panel) and aged animals (right panel). Note that tetanization resulted in larger left-ward shift in I-O curves in young compared to aged animals. Number in brackets depict number of slices compared. 
region and elicited by stimulation of Schaeffer collaterals with increasing stimulus intensity. The slope of fEPSPs mirroring strength of basal glutamatergic synaptic transmission in acute brain slices of young animals was not different from that in aged animals $\left(F_{(2,335)}=0.36, p\right.$ $=0.98 ; n=$ at least 10 slices, Figure $1 \mathrm{~A})$. Consequently, comparison of fEPSPs plotted against the fiber volley amplitude yielded analogous results (Figure 1A). Subsequently, we compared short-term synaptic plasticity in both groups by measuring the rate of fEPSP slope facilitation in response to paired stimulation (paired pulse facilitation, PPF; inter-stimulus interval $50 \mathrm{~ms}$ ). We found no significant difference in PPF index in young compared to aged animal slices (PPF index was $1.58 \pm 0.04$ and 1.55 \pm 0.07 in young and aged animals, respectively, $n=$ at least 10 slices, $p=0.64$, Figure 1B).

Next, we assessed the impact of age on LTP characteristics. To this end we applied high frequency stimulation (HFS, 4 x $100 \mathrm{~Hz}$ ), setting the stimulation current at the value yielding approx. 30 - $40 \%$ max. slope of fEPSP before HFS. In young animals, HFS resulted in a post-tetanic potentiation (PTP) that reached 206.1 \pm 14.07 $\%$ of basal fEPSP slope ( $n=8$ slices, $p<0.01$ compared to baseline, Figure 1C) which subsequently declined reaching a new steady value of $160.2 \pm 8.45 \%$ of the baseline at 90 minutes after LTP induction $(n=8, p<0.01$ compared to baseline, Figure $1 \mathrm{C})$. In aged animals, average PTP was $195.06 \pm 22.39 \%$ of basal signal $(n=8, P<0.01$ compared to baseline but not statistically different from PTP in young animals, $p=0.22$ ) after which the signal decreased and stabilized attaining the value of $126.8 \pm 7.3$ $\%$ of basal fEPSP slope ( $90 \mathrm{~min}$ post HFS, $n=8, p<0.01$ of baseline, Figure 1C). Following PTP, the magnitude of fEPSPs potentiation recorded in young animals was significantly larger than in aged animals throughout the time of recording ( $p<0.001$ for time window of 5-90 minutes, unpaired student $t$-test, Figure 1C). In young animals, I-O relationship for fEPSP slope analyzed 90 min. post LTP induction revealed a significant upward shift $\left(F_{(2,255)}=4.137, p<0.001\right.$, Figure 1D). Similarly, in aged animals, upward shift was also observed $\left(F_{(2,25)}=1.703, p\right.$ $<0.05$, Figure 1D). We additionally monitored PPF ratio 90 minutes post LTP induction and compared its value with those recorded before LTP in basal conditions. In slices from young animals, LTP magnitude at 90 minutes post induction significantly correlated with reduction of PPF ratio (relative PPF change was $0.90 \pm 0.019, n=$ 8 , Pearson correlation coefficient $-0.628, p<0.01$, data not shown). Similarly, in aged animals, LTP magnitude correlated with PPF ratio reduction (relative PPF change was $0.91 \pm 0.04, n=8$, Pearson correlation coefficient $-0.669, p<0.05$, data not shown). In conclusion, slices of both young and aged animals did not significantly differ in viability and basal synaptic transmission within considered time window but the extent of LTP was significantly smaller in aged group for most of the recording time.
Moreover, successful induction of LTP correlated with respective shift in I-O curves and PPF reduction.

We next checked the impact of glycogen phosphorylase blockade (5 $\mu \mathrm{M}$ BAY) on synaptic transmission in the Sch-CA1 pathway in young and aged animals. We found that thirty to ninety minutes of drug application did not significantly affect fEPSP slope recorded in slices from both young and aged animals upon basal stimulation (fEPSP relative change was 0.98 \pm 0.01 and $0.99 \pm 0.02$, respectively, $n=8, p>0.7$, data not shown). Moreover, PPF ratio in the presence of BAY was not altered in both groups investigated (PPF ratio was $1.53 \pm 0.05$ and $1.46 \pm 0.04$ in slices of young and aged animals, respectively; $n=8, p=0.47$ and $p=0.314$ compared to respective controls, data not shown). Thus, BAY did not affect basal synaptic transmission or shortterm facilitation.

We then considered the impact of BAY on synaptic plasticity. In young animals, HFS applied in the presence of BAY resulted in similar PTP $(195.9 \pm 11.1 \%$ of baseline, $n=8, p=0.1$ compared to untreated controls) but LTP maintenance phase was completely disrupted (LTP at 90 minutes post induction was $93.3 \pm 13.6 \%$ of baseline, $p<0.01$ compared to untreated controls, $n$ $=8$ slices, Figure 2A). Moreover, I-O curves generated before and 90 minutes post LTP induction in BAY treated slices were not significantly shifted $\left(F_{(2,256)}=0.340, p=\right.$ 0.99 , Figure $2 \mathrm{~B}$, significantly different compared to I-O curves in control post LTP $\left.F_{(2,256)}=2.896, \mathrm{p}<0.001\right)$ and PPF ratio, following HFS, did not significantly change (relative PPF ratio change was $1.03 \pm 0.02, p=0.29$, data not shown).

Next we repeated this experimental paradigm for slices from aged animals. Surprisingly, we found that in the presence of BAY, PTP was significantly larger than in control aged group (fEPSPs slope potentiation was $262.69 \pm 15.2 \%$ of basal signal, $p<0.05$ relative to aged controls, $n=8$, Figure 2C). Following PTP, the magnitude of fEPSPs potentiation recorded in aged animals in the presence of BAY was significantly larger than in aged controls in the first 60 minutes following HFS ( $p<0.001$, unpaired student $t$-test, Figure 2C). After that time LTP magnitude in the presence of BAY, although on average larger, was not significantly different from that recorded in aged controls (65-90 min., $p>0.05$, Figure $2 \mathrm{C})$. In the same set of preparations, we observed a significant upward shift in I-O relationship for fEPSP slope analyzed $90 \mathrm{~min}$ post LTP $\left(F_{(2,256)}=2.274, p=0.004\right.$, Figure 2D). This upward shift was significantly larger than that observed in control slices of aged animals $\left(F_{(2,256)}=1.450, p=\right.$ 0.045 , most evident for strong stimuli of $200 \mu \mathrm{A}-300 \mu \mathrm{A}$, Figure 2D). In slices from aged animals treated with BAY, basal PPF ratio was $1.46 \pm 0.04$ and was not significantly different from the respective control $(p=0.314, n=8$, data not shown). After LTP induction, average PPF ratio was reduced to similar extent as in controls (relative PPF ratio 
change 90 minutes post HFS was $0.91 \pm 0.03, p=0.89$ compared to controls, data not shown). Altogether, unlike young animals, pretreatment of slices from aged animals with phosphorylase inhibitor BAY did not interfere with the magnitude of LTP in Sch-CA1 projection showing even a trend to increase it.
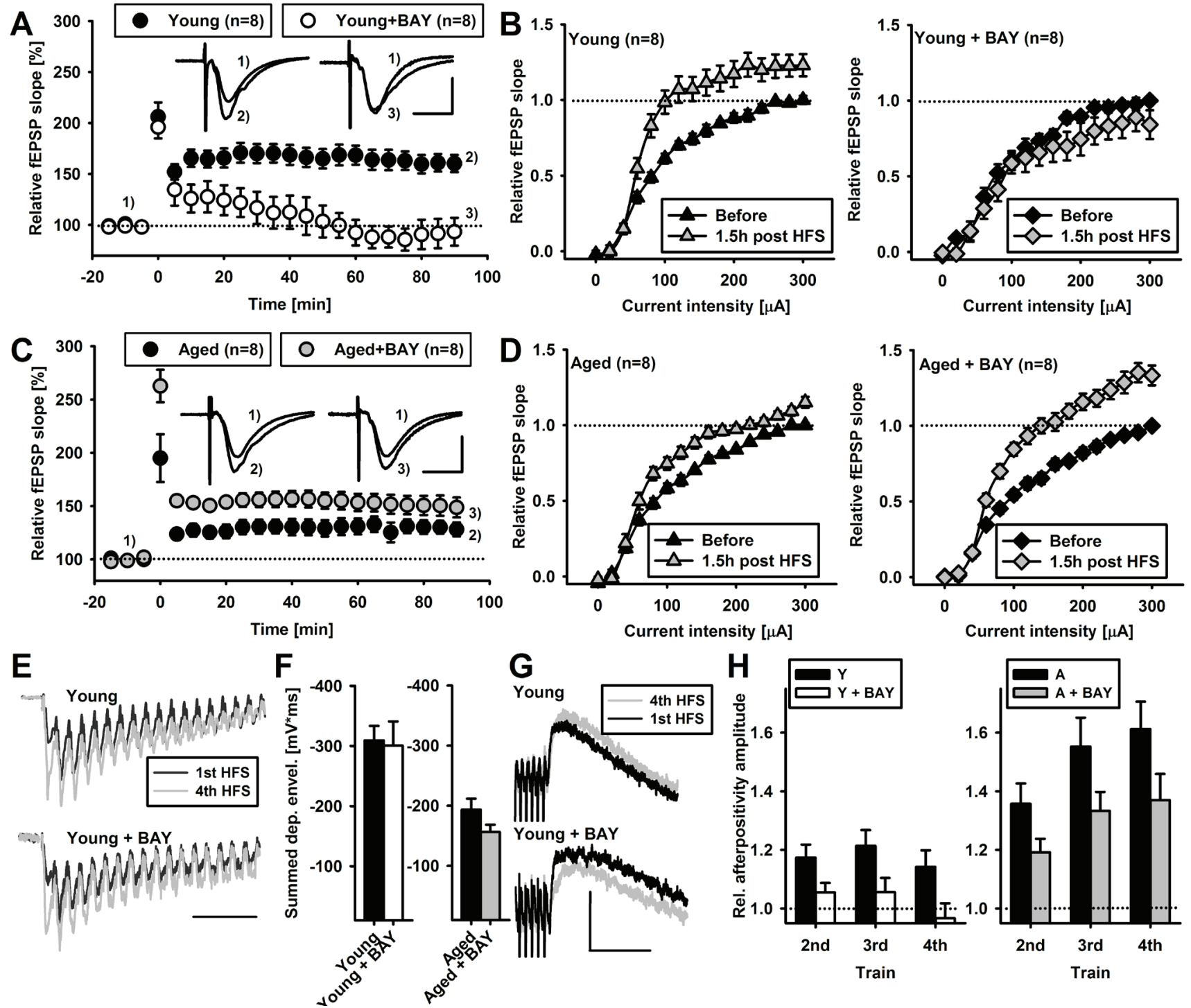

Figure 2: Glycogen phosphorylase inhibitor, BAY U6751, differentially affects LTP in young and aged animals. A. Average time-course of fEPSP slopes recorded in young animals in the absence (black circles) or presence of bath applied inhibitor (white circles) before and after tetanization $(4 \times 100 \mathrm{~Hz}$, time 0 minutes) normalized to baseline values. Insets show exemplary fEPSPs recorded before (1) and 90 minutes (2,3) after tetanization (scale: $0.4 \mathrm{mV}, 10 \mathrm{~ms}$ ). Note that bath application of BAY (10 $\mu \mathrm{M})$ completely abolished LTP in young animals. B. Average fEPSP slopes plotted against current stimulus (I-O curves) before (black) and 90 minutes post tetanization (grey) in young animals in the absence (left panel) or presence of inhibitor (right panel). Note that BAY application completely abolished left-ward shift of I-O curve observed in controls. C.-D. The same experiments presented in A-B performed in slices of aged animals. Note that bath application of BAY did not abolish but rather enchanced LTP magnitude in aged animals (C). Note that bath application of BAY enchanced left-ward shift of I-O curve compared to aged controls. E. Exemplary traces of initial fEPSPs recorded in young animals during first (black trace) and fourth (grey trace) high frequency stimulation (HFS, $100 \mathrm{~Hz}$ ) in the absence (upper panel) and in the presence of bath applied inhibitor (lower panel). Note that BAY does not significantly alter the time-course of fEPSPs during HFS (scale: $50 \mathrm{~ms}$, amplitudes were normalized). F. Average depolarization envelopes for all four trains of stimuli in young (left panel) and aged (right panel) animals. Note that in the presence of inhibitor (BAY), average depolarization envelopes did not significantly change. G. Exemplary traces of fEPSPs time-course recorded immediately following cessation of HFS in young animals (black traces). Note the positive deflection occurring immediately following HFS (afterpositivity, AFP). Panels show exemplary trace of AFP recorded following first (black trace) and fourth (grey trace) in the absence (upper panel) and in the presence (lower panel) of the inhibitor (scale: $0.15 \mathrm{mV}$, $100 \mathrm{~ms}$ ). H. Average AFP amplitude following subsequent trains of HFS normalized to AFP of the first train in young (left panel) and aged animals (right panel). Note that BAY application significantly reduced the scaling of AFP amplitude in both young and aged animals $(P<$ 0.05 , ANOVA). Number in brackets depict number of slices compared. 
Divergent effects of BAY administration on post-tetanic potentiation and, subsequently, on LTP magnitude in young and aged animals could indicate altered conditions of LTP induction. To get an insight into phenomena occurring upon stimulation, we next analyzed fEPSPs during trains of HFS. In slices from young animals, average depolarizing fEPSP envelope during the $1^{\text {st }}$ train was $-55.33 \pm 3.86 \mathrm{mV}^{*} \mathrm{~ms}$ and it was not changed upon bath application of the drug $(-52.88 \pm 5.79 \mathrm{mV} * \mathrm{~ms}$, $p=0.7, n=8$, data not shown). Summed depolarizing envelope following four, one second long, HFS trains was $-309.2 \pm 24.1 \mathrm{mV}^{*} \mathrm{~ms}, n=8$, Figure $2 \mathrm{~F}$ ), and this parameter was not changed upon bath application of BAY $\left(-300.45 \pm 40.3 \mathrm{mV}^{*} \mathrm{~ms}, n=8, p=0.72\right.$, Figure $\left.2 \mathrm{~F}\right)$. Analogous analysis performed on data from aged animals revealed that average depolarizing fEPSP envelope during the $1^{\text {st }}$ train was significantly smaller compared to young animals $\left(-40.1 \pm 4.2 \mathrm{mV}^{*} \mathrm{~ms}, n=8, p=0.019\right.$ compared to young animals) and it was not changed upon bath application of BAY $\left(-37.47 \pm 4.81 \mathrm{mV}^{*} \mathrm{~ms}, p=0.7\right.$, $n=8$, data not shown). Summed depolarizing envelope following four HFS trains was $-193.4 \pm 18.4 \mathrm{mV}^{*} \mathrm{~ms}, n$ $=8$, Figure $2 \mathrm{~F}$ ), and this parameter was not significantly different upon bath application of BAY $(-156.3 \pm 12.3$ $\mathrm{mV} * \mathrm{~ms}, n=8, p=0.09$, Figure 2F).

Next we analyzed the amplitude of afterpositivity (AFP) that peaked approximately $50-100 \mathrm{~ms}$ after the cessation of HFS (Figure 2G). In slices of young animals, average afterpositivity amplitude occurring following $1^{\text {st }}$ HFS was $0.19 \pm 0.01 \mathrm{mV}(n=8)$ and it was not different in slices treated with BAY $(0.23 \pm 0.02, n=8, p=0.22$, data not shown). Following repeated HFS stimulation, afterpositivity amplitude varied with each subsequent HFS (relative AFP amplitude normalized to $1^{\text {st }}$ train value was $1.17 \pm 0.04,1.21 \pm 0.05$ and $1.14 \pm 0.05$ for $2^{\text {nd }}-4^{\text {th }}$ train, respectively, $n=8$, Figure $2 \mathrm{H}$ ). In the presence of BAY, the increment of AFP amplitude was significantly attenuated (relative AFP amplitude in the presence of $\mathrm{BAY}$ was $1.05 \pm 0.03,1.05 \pm 0.04$ and $0.96 \pm 0.05$ for $2^{\text {nd }}-4^{\text {th }}$ train, respectively, $F_{(1.48)}=14.326, n=8, p<0.001$ compared to untreated controls, Figure $2 \mathrm{H}$ ).

In slices of aged animals, average afterpositivity amplitude occurring following $1^{\text {st }}$ HFS was $0.17 \pm 0.02$ $\mathrm{mV}(n=8$, not significantly different from slices of young animals, $p=0.28$ ) and it was not different in slices treated with BAY $(0.16 \pm 0.01, n=8, p=0.69$, student $t$-test, data not shown). With each subsequent HFS train, relative AFP amplitude increased (relative afterpositivity amplitude was $1.35 \pm 0.06,1.55 \pm 0.09$ and $1.61 \pm 0.09$ following $2^{\text {nd }}-4^{\text {th }}$ train, respectively, $n=8$, Figure $2 \mathrm{H}$ ). In the presence of BAY, the increment of AFP amplitude was significantly attenuated (relative afterpositivity amplitude in the presence of BAY was $1.19 \pm 0.04,1.33 \pm 0.06$ and $1.36 \pm 0.08$ for $2^{\text {nd }}-4^{\text {th }}$ train, respectively, $F_{(1,48)}=6.857$, $n=8, p=0.01$ compared to untreated controls, Figure $2 \mathrm{H})$. In conclusion, BAY had no impact on depolarizing envelope during LTP induction, but attenuated HFSinduced changes in afterpositivity amplitude in both young and aged groups.

\section{mRNA expression profile depends on the electrophysiological stimulation and the animal age}

In slices that were subjected to electrophysiology recordings, we checked the correlation between neuronal activity and the mRNA expression for several metabolic enzymes in neurons and astrocytes $(n$ indicates number of analyzed individual cells, see Materials and Methods; Figure 3). As a reference gene we used glutamine synthetase (Glns), an astrocyte-specific enzyme, which catalyzes the condensation of glutamate and ammonia to form glutamine. Gln protein in neurons is practically undetectable and its expression is activated only in absence of astrocyte e.g. in. pure neuronal culture [12]. We found that 90 minutes after LTP induction, astrocytic mRNA for Glns significantly increased in both young and aged animals (respectively $794 \% \pm 31, n=2040$ and $760 \% \pm 8, n=1965$ compared to non-stimulated tissue samples, $p<0.01$, Figure 3I, 3L). Moreover, the basal (90min, $0.1 \mathrm{~Hz}$ ) exogenous stimulation also led to increase in mRNA for Glns in astrocytes $(433 \% \pm 38, n=2505$ and $523 \% \pm 9, n=2011$ for young and aged rats respectively). Simultaneously, we could not observe any increase in mRNA for Glns in young rat's neurons. It is noteworthy that the level of Glns mRNA after exogenous stimulation was significantly higher in neurons of aged rats compared to the young animals (Figure 3I vs 3L).

Next, we checked the mRNA expression for brain isozyme of glycogen phosphorylase (Pygb) and muscle isoform of glycogen synthase (Gys1) and we found that basal stimulation $(90 \mathrm{~min}, 0.1 \mathrm{~Hz}$ ) significantly elevated mRNA expression for both enzymes in astrocytes (in young as well as in aged rats) as compared to unstimulated hippocampal sections but in the case of neurons the increase was restricted only to the aged animals (Figure 3J$3 \mathrm{~K}$ and Figure 3M-3N). Evidently, exogenous stimulation of the Sch-CA1 pathway resulted in statistically significant increase in mRNA level for key glycogen metabolism enzymes in astrocytes.

Interestingly, we found that even basal stimulation (90min, $0.1 \mathrm{~Hz}$ ) of the slices was sufficient for the increase of Pygb and Gys 1 level in astrocytes $(296 \% \pm 22, n=$ 3101 and $734 \% \pm 16, n=2682$ respectively for $P y g b$ and Gysl in young rats and $636 \% \pm 21, n=2184$ and $176 \%$ $\pm 20, n=2447$ respectively for Pygb and Gys 1 in aged rats). Further stimulation using $4 \times 100 \mathrm{~Hz}$ train did not strengthen the effect on mRNA level in young and aged astrocytes $(287 \% \pm 19, n=2253$ and $696 \% \pm 13, n=$ 2747 respectively for $P y g b$ and Gys 1 in young rats; 602 $\% \pm 13, n=2858$ and $270 \% \pm 18, n=1908$ respectively 
for Pygb and Gysl in aged animals, Figure 3J-3K and Figure $3 \mathrm{M}-3 \mathrm{~N}$ ). As it is shown in Figure 3, mRNA up-regulation for key glycogen metabolism enzymes occurred in astrocytes in both age groups. On the other hand, the changes in the mRNAs expression in neurons showed a different pattern. In young animals, we observed relatively constant level of Pygb in neurons regardless of electrophysiological treatment (C vs CS vs LTP, Figure 3J) whereas, for Gys1 we found a doubling of mRNA expression following basal stimulation and LTP induction as compared to its initial level in hippocampal slices (CS vs $\mathrm{C}=261 \% \pm 15$, LTP vs $\mathrm{C}=232 \% \pm 10$, Figure $3 \mathrm{~K}$ ). In contrast to young rats, neurons of aged animals exhibited significantly higher levels of mRNA for Pygb in CS (316
$\% \pm 15, \mathrm{n}=1157)$ and LTP slices $(231 \% \pm 5, n=1314 \mathrm{see}$ Figure 3M) and for Gys1 as well (CS vs C = 509 \% \pm 10 ; LTP vs $\mathrm{C}=416 \% \pm 6$, Figure $3 \mathrm{~N}$ ).

In conclusion, mRNA level for key metabolic enzymes (Gys1, Pygb and Glns) is differentially regulated by exogenously evoked synaptic activity in Sch-CA1 region of young and aged rat hippocampal slices.

\section{Impact of glycogen phosphorylase inhibitor on mRNA expression level of metabolic enzymes}

Next, we repeated the above described FISH analysis in slices in which LTP was evoked in the presence of glycogen phosphorylase inhibitor BAY. With regard to
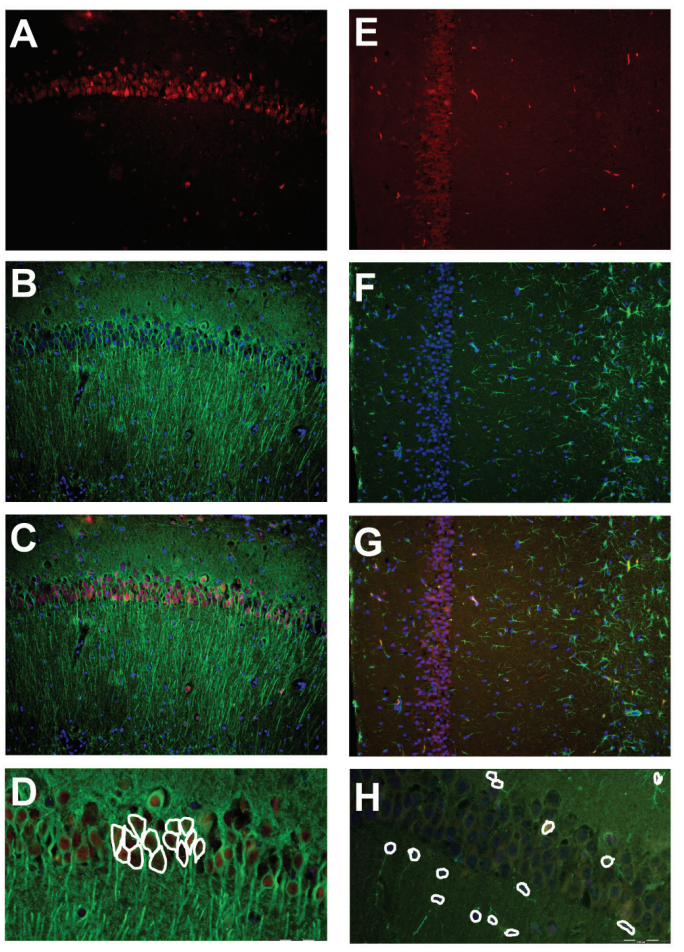
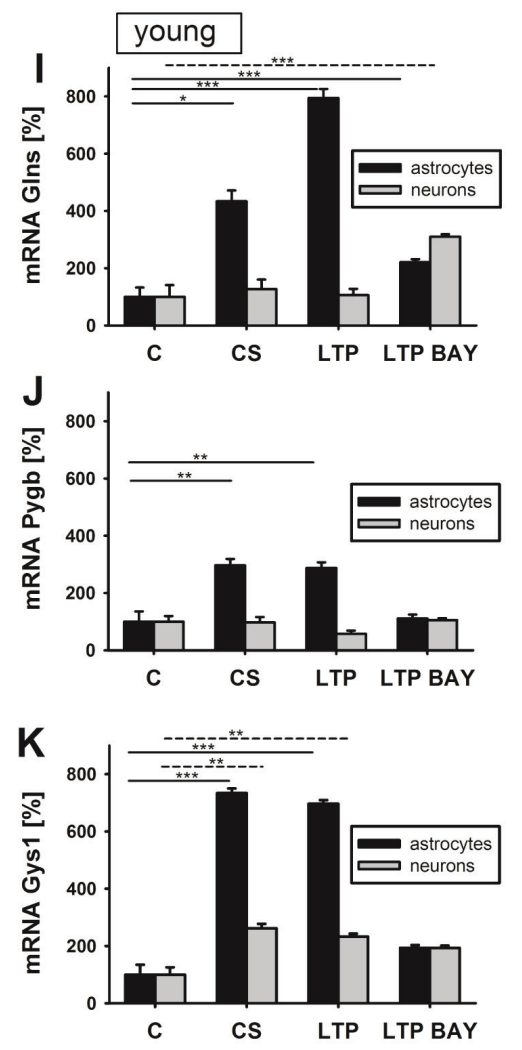
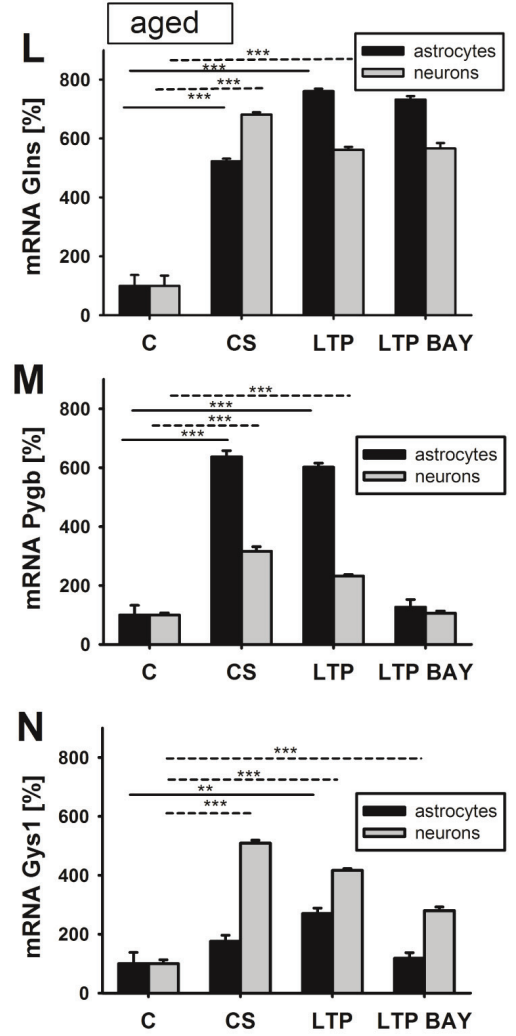

Figure 3: Effect of glycogen phosphorylase (Pygb) inhibition on mRNA expression of key metabolic enzymes following LTP depends on cell type and age. A.-H. Localization of mRNA for Pygb in hippocampal slices of young rats 90 minutes following LTP induction. A. and E. FISH staining against Pygb; B. and F. IF staining against MAP-2 (B) or GFAP (F); C. merged A+B; G. merged E+F. D. exemplary analysis of a population of pyramidal cells (CA1 hippocampal region) or astrocytes (H) with marked region of mRNA expression analysis. I.-N. Statistics of the effect of glycogen phosphorylase inhibition with BAY U6751 on the basal and HFS stimulationinduced changes in the expression of mRNA for glutamine synthetase (Glns), Pygb or glycogens synthase 1 (Gys1) in young (I-K) or aged (L-N) rat hippocampi. mRNA expression analysis performed for basal stimulated (CS), LTP-induced (LTP) and LTP-induced in the presence of BAY (LTP BAY) samples. The expression in unstimulated control (C) was normalized to $100 \%$. Average number of analyzed cells in a probe was $n=2282$ and $n=1049$ for astrocytes and neurons, respectively. The $p$ values (asterisks) for astrocytes (black bars) and neurons (grey bars) were as follows: I) CS $v s$ C - 4.92E-02 and 0.64; LTP $v s$ C - 1.34E-02 and 0.95; LTP BAY vs C - 4.63E-05 and 2.59E-05. J) mRNA for Pygb: CS $v s$ C - 9.82E-03 and 0.9; LTP $v s$ C - 7.20E-03 and 5.07E-02; LTP BAY vs C - 0.75 and 0.85. K) mRNA for Gys1: CS vs C - 9.47E-06 and 1.59E-03; LTP vs C - 2.88E-07 and 8.48E-04; LTP BAY vs C - 5.00E-04 and 3.97E-02; L-N for aged rats L) mRNA for Glns: CS vs C - 4.03E-08 and 4.64E-08; LTP vs C - 1.32E-09 and 4.48E-06; LTP BAY vs C - 1.61E-07 and 6.73E-04. M) mRNA for Pygb: CS vs C - 3.51E-03 and 7.35E-04; LTP vs C - 3.3E-0402 and 2.24E-04; LTP BAY vs C - 0.71 and 0.8. N) mRNA for Gys1: CS vs C - 0.14 and 1.07E-05; LTP vs C - 9.56E-03 and 1.06E-07; LTP BAY vs C - 0.67 and 2.47E-04. 
Glns mRNA expression, we found that in astrocytes of young rats, mRNA level of this enzyme was significantly lower compared to LTP slices in the absence of the drug $(\mathrm{LTP}=794 \% \pm 31, n=2040 v s$ LTPBAY $=221 \% \pm 10$, $n=1519$ Figure 3I). In contrast, BAY treatment had no impact on Glns mRNA expression in astrocytes or neurons in aged animals (LTP BAY astrocytes $=732 \% \pm 11, n$ $=1193$ and LTP BAY neurons $=566 \% \pm 18, n=1212$, Figure 3L). Next, we investigated the mRNA expression for Pygb and Gys1. We found that treatment of slices with BAY prevented the up-regulation of mRNA expression for Pygb and Gys 1 in astrocytes and neurons of both young and aged animals (Figure 3J, 3M and Figure 3K, 3N). Thus, in the presence of glycogen phosphorylase inhibitor, mRNA up-regulation following LTP induction is lost regardless of magnitude of LTP (see Figure 2A and 2C)

\section{Beta amyloid spots are absent in young and appear in tissue of aged animals}

To see age-related changes in the structure of hippocampal region we stained the slices using antibodies against beta-amyloid peptide for both age groups of animals ( $\mathrm{n}$ for each $=4$ ). We found that in the considered here Sch-CA1 projection, the beta-amyloid peptide was present in all slices from aged animals hippocampi and was virtually absent in those from young rats. Slightly unexpectedly, in the former group, it was localized mainly inside cells and, to a lesser extent, in the extracellular space. Moreover, the slices from aged animals showed reduced neuron density and distorted geometry of hippocampal area as compared to the young ones (Figure 4).
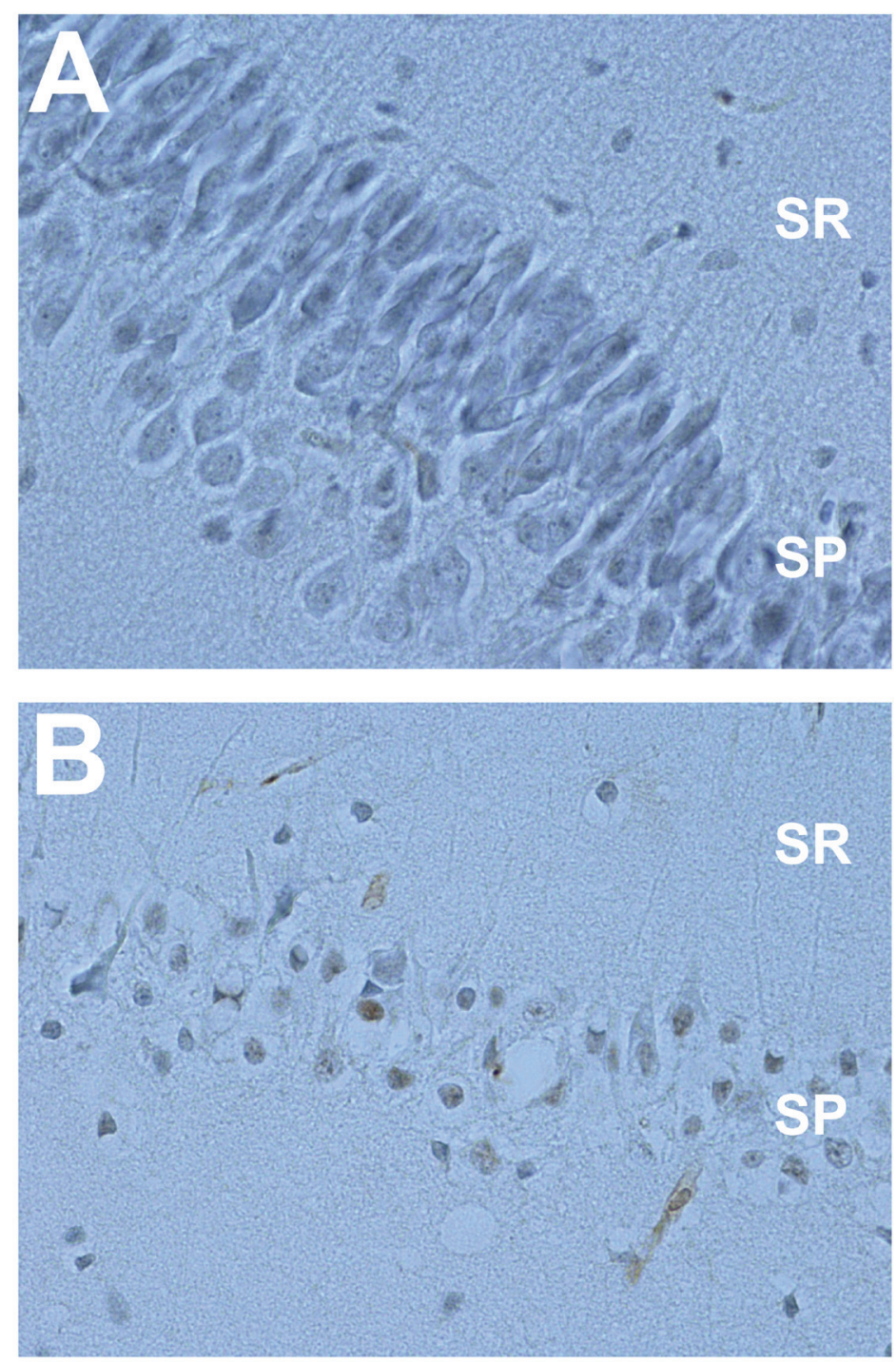

Figure 4: Aging-related changes in hippocampal area are associated with the appearance of beta amyloid spots and distorted geometry of neuronal network. Immunochemical staining against beta-amyloid peptide in CA1 hipocampal region of A. young rat and $\mathbf{B}$. aged rat. SR, stratum radiatum; SP, stratum piramidale. 
Table 1: Cy3 fluorescence probes used for FISH analysis.

\begin{tabular}{|c|c|c|c|}
\hline & enzyme & $\begin{array}{c}\text { Protein } \\
\text { symbol }\end{array}$ & Oligonucleotide sequence anti-mRNA 5'-3, \\
\hline 1 & $\begin{array}{c}\text { glycogen } \\
\text { synthase type 1 }\end{array}$ & Gys (Gys1) & GGGTCCCCCCTCGATCAGCCAACGCCCAAAATACACCTTA \\
\hline 2 & $\begin{array}{c}\text { glycogen } \\
\text { phosphorylase brain }\end{array}$ & Pygb (Pygb) & AGGGTGGCGGCCACCACAAAGTACTCCTGCTTCAGCCGCA \\
\hline 3 & glutamine synthetase & Glns (Glns) & GCTTGATGCCTTTGTTCAAGTGGGAACTTGCTGAGGTGGC \\
\hline
\end{tabular}

Table 2: Categories of hippocampal slices prepared in electrophysiology experiments for the dendritic spine morphological analysis.

\begin{tabular}{|c|c|c|}
\hline $\begin{array}{c}\text { hippocampal } \\
\text { slice name }\end{array}$ & abbreviation & electrophysiological stimulation \\
\hline C & Control in ACSF buffer & No \\
\hline C BAY & $\begin{array}{c}\text { Control in ACSF buffer+5uM } \\
\text { BAY }\end{array}$ & No \\
\hline CS 30 & ACSF buffer & 30 min of basal stimulation \\
\hline LTP 30 & ACSF buffer & 30 min after LTP induction \\
\hline CS BAY 30 & ACSF buffer+5uM BAY & 30 min of basal stimulation with BAY \\
\hline LTP BAY 30 & ACSF buffer+5uM BAY & 30 min after LTP induction with BAY \\
\hline CS 90 & ACSF buffer & 90 min of basal stimulation \\
\hline LTP 90 & ACSF buffer & 90 min after LTP induction \\
\hline CS BAY 90 & ACSF buffer+5uM BAY & 90 min of basal stimulation with BAY \\
\hline LTP BAY 90 & ACSF buffer+5uM BAY & 90 min after LTP induction with BAY \\
\hline
\end{tabular}

The effect of plasticity induction, glycogen phosphorylase inhibition and aging, on dendritic spines morphology

In order to investigate age dependent effect of glycogen phosphorylase blockade on structural plasticity of dendritic spines we analyzed dendritic spine morphology in secondary/ternary dendrites of CA1 hippocampal region. To this end, neurons were stained with a lipophilic dye (DiI) then length and head width of dendritic spine were measured. Thus we were able to calculate further a scale-free parameter length-to-width ratio, which effectively describes the spine shape.

First, to check whether BAY treatment affects the morphological parameters of dendritic spines or whether the basal stimulation influence the spine shape characteristic, we did a number of comparisons between control (unstimulated) samples (Table 3). In none of mentioned juxtapositions we found any difference between animal groups in terms of BAY presence and basal stimulation (Table 3 ). Thus we conclude that all analyzed animal groups were equivalent for further analysis of HFS induced changes in spine morphology.

It is well established that LTP induction alters spine morphology. Since, as shown herein, the extent of LTP was markedly affected by Pygb blockade (by BAY) in an age-dependent manner, we were seeking to find a correlation between these functional data and dendritic spine morphology. In order to assess the impact of BAY on dendritic spines after LTP induction, we compared spine geometry at $30 \mathrm{~min}$ and $90 \mathrm{~min}$ after HFS stimulation vs control groups (basal stimulation at the same time points). Major trends observed based on results of our analysis are summarized in the Table 4.

We analyzed length-to-width ratio (i.e. the length divided by the width), which reflects the spine shape and thus effectively describes the spine form. Decrease in these parameters shows change in shapes towards being shorter and wider, thus being more like mushroom spines that are indicated as more matured. We showed statistically significant decrease in the length-to-width parameter after LTP in both young and aged rats CS 90 vs LTP 90 (young: $2.87 \pm 0.36$ ( $n=7$ slices $) v s 1.84 \pm 0.24$ ( $n=12$ slices $)$, p-value t-test, $p<0.005$, aged: $3.30 \pm 0.51$ ( $n=15$ slices $)$ vs $2.35 \pm 0.28$ ( $n=8$ slices), $p$-value t-test $p<0.005$ ). Similar differences in the length-to-width parameter of spines occurred in presence of BAY in both young and aged rats CS BAY $90 v s$ LTP BAY 90 (young: $2.92 \pm 0.32$ ( $n=12$ slices) vs $2.21 \pm 0.34$ ( $n=11$ slices), $p$-value $t$-test, $p<0.005$, aged: $3.30 \pm 0.47$ ( $n=19$ slices) vs $2.43 \pm 0.26$ ( $n=22$ slices), $p$-value $t$-test $p<0.005$ ).

Because the individual subpopulations of spines might determine the structural plasticity upon HFS we divided the spines into subpopulations according to the length-to-width parameter (Figures 5A, 5D and $6 \mathrm{~A}, 6 \mathrm{D})$. To provide a detailed description of the spine shape dynamics upon stimulation we analyzed agedependent changes of individual bins of length-to-width distribution both in the presence and absence of BAY. This approach led to the identification of spine groups that displayed different dynamics of morphological changes. 
We found that BAY treatment restrained HFS-induced maturation of spines in young rats. The statistically significant differences were found solely in two bins of the distribution (Figure 5D). More prominent changes of shape parameters (toward mushroom shaped spines i.e. lower values of length-to-width) were observed in spines of aged rats (Figure 6D). The analysis of spine shape (Figures 5B, 5E and 6B, 6E) and cumulative distributions of shape parameter (Figures $5 \mathrm{C}, 5 \mathrm{~F}$ and $6 \mathrm{C}, 6 \mathrm{~F}$ ) further support this observation (cf. Figure $5 \mathrm{~F}$ and Figure $6 \mathrm{~F}$ ). The maximum difference (D) between cumulative curves for young and aged animals were found to be: $\mathrm{D}=0.26$ for CS 90 vs LTP 90, D = 0.26 for CS BAY 90 vs LTP BAY 90 and $\mathrm{D}=0.39$ for CS 90 vs LTP 90, D = 0.29 for CS BAY 90 vs LTP BAY 90, respectively.

Finally, we analyzed the aged dependent effect of inhibition of glycogen degradation on density of dendritic spines of interest. The incubation with BAY did not affect the total density of dendritic spines upon HFS induced LTP (Table 5).

\section{DISCUSSION}

In this paper we demonstrate that the inhibition of glycogen breakdown in astrocytes has an opposite effect on the LTP magnitude in young and aged rats, in young group it disrupts LTP maintenance whereas in the aged ones it appears to favor elevated extent of LTP. Importantly, alteration in LTP dependence on glycogen phosphorolysis in young and aged groups are corroborated by observation of different modes of dendritic spines maturation in young and aged animals (Figure 5, 6). Evidently, the

\section{young}

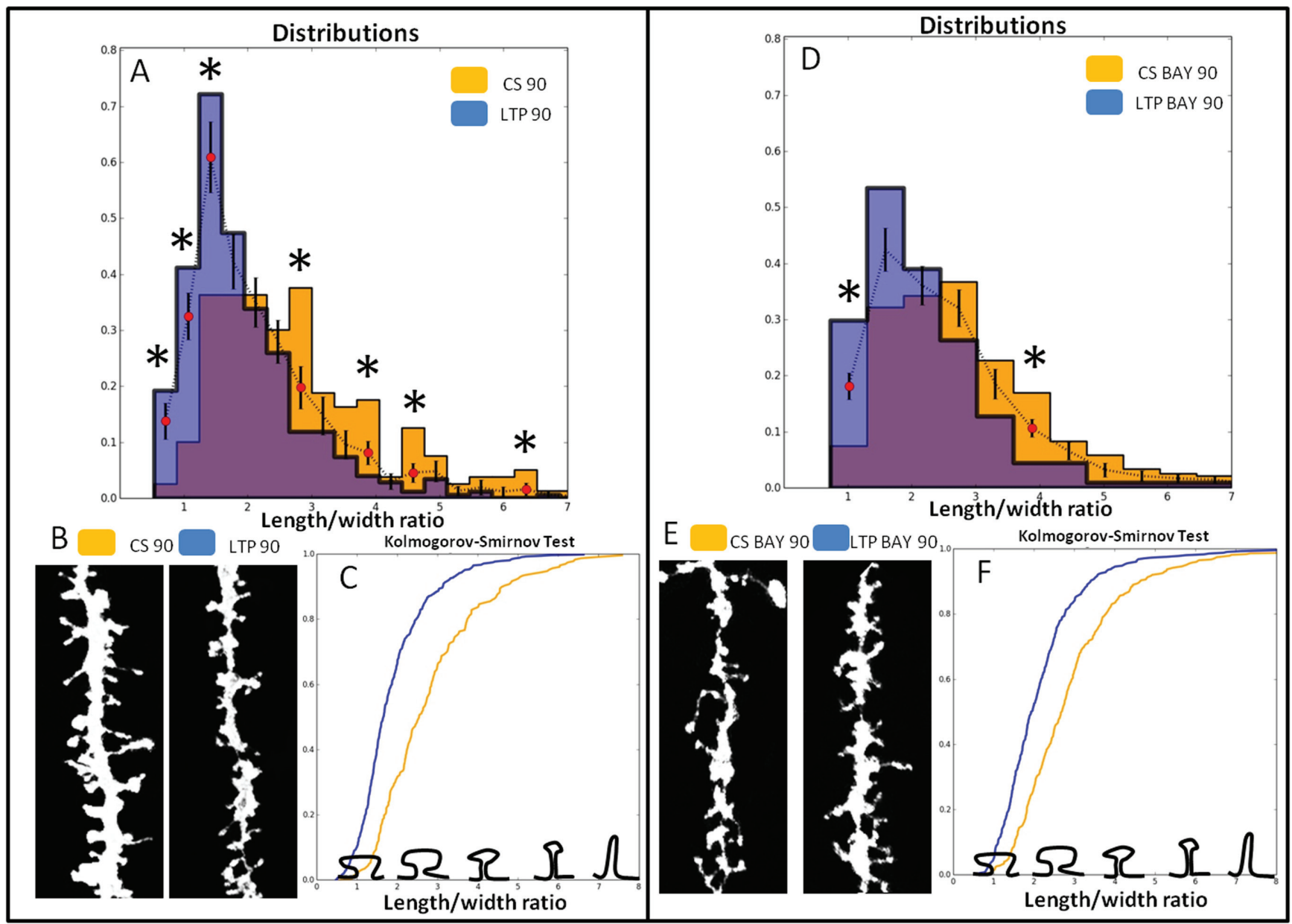

Figure 5: Glycogen phosphorylase inhibitor, BAY U6751, affects HFS induced dendritic spine morphology in young animals. A. and D. Distribution of dendritic spines shape parameter (length-to-width) in LTP-induced (LTP 90) and LTP-induced in the presence of BAY (LTP BAY 90) samples in respect to the basal stimulated controls at 90 min (CS 90 and CS BAY 90 respectively). (*) indicates bins with statistically significant differences. B. and E. Examples of DiI-stained neurons in the CA1 area of rat hippocampus (pictures present secondary apical dendrites). C. and F. Cumulative frequency of the shape parameter (length/width) of spines in control and after LTP induction in brain slices. Kolmogorov-Smirnov test revealed significant differences in the spine shape parameters of CS 90 -compared with LTP 90 and CS BAY 90 compared with LTP BAY $90(p<0.001)$. 
inhibition of glycogen degradation suppressed HFSinduced spine maturation in young animals, whereas the opposite was seen in aged rats. The most intriguing question emerging from these findings concerns agerelated changes in the astrocytic metabolism supporting the synaptic plasticity. Although, lactate produced by astrocytes from extracellular glucose is known to be the main energy substrate for neurons [13, 14], it was proposed that glycogen-derived, but not glucose-derived, lactate is a prerequisite for the plasticity phenomena in neonatal and young animals [15]. In agreement with this scenario, it was also shown that inhibition of glycogen breakdown and lactate release from astrocytes disrupted memory formation in hippocampus $[8,16]$ and impaired LTP [8]. It seems thus that indeed, a key to understand the mechanism underlying our observations lies in differential roles of glycogen phosphorolysis and their consequences in neuronal-astrocytic cross-talk. Importantly, upon basal stimulation (BS), there were no differences between fEPSPs measured in aged and young groups and, in these conditions, BAY had no effect. Thus, glycogen phosphorolysis appears to affect mainly the synaptic plasticity rather than the basal activity. Depolarizing envelope measured during LTP induction was not affected by BAY but BAY treatment significantly attenuated relative changes in afterpositivity (AFP) amplitude following HFS in both age groups. Afterpositivity occurring following intense neuronal activity is believed to primarily mirror activity of postsynaptic calcium- and voltage-dependent potassium conductances (e.g [17-20]) which play an important role in LTP, learning and memory [21-23]. Decreased dynamics of AFP in subsequent HFS

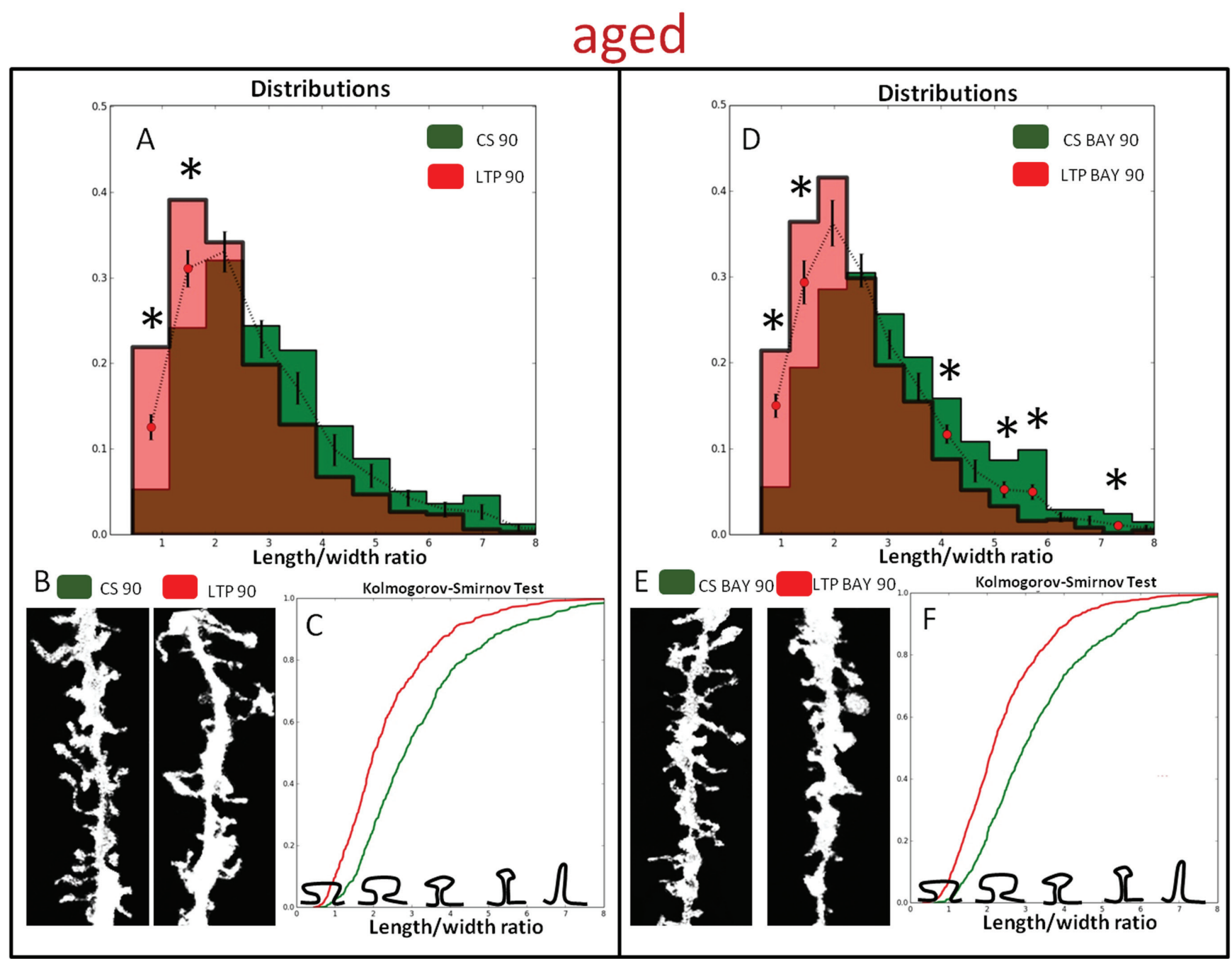

Figure 6: Glycogen phosphorylase inhibitor, BAY U6751, affects HFS induced dendritic spine morphology in aged animals. A. and D. Distribution of dendritic spines shape parameter (length-to-width) in LTP-induced (LTP 90) and LTP-induced in the presence of BAY (LTP BAY 90) samples in respect to the basal stimulated controls at 90 min (CS 90 and CS BAY 90 respectively). (*) indicates bins with statistically significant differences. B. and E. Examples of DiI-stained neurons in the CA1 area of rat hippocampus (pictures present secondary apical dendrites). C. and F. Cumulative frequency of the shape parameter (length/width) of spines in control and after LTP induction in brain slices. Kolmogorov-Smirnov test revealed significant differences in the spine shape parameters of CS 90 -compared with LTP 90 and CS BAY 90 compared with LTP BAY $90(p<0.001)$. 
Table 3: Dendritic spine morphology of young and aged rats.

\begin{tabular}{|c|c|c|c|c|c|}
\hline increase & $\uparrow$ & \multirow{3}{*}{ young } & increase & $\uparrow$ & \multirow{3}{*}{ aged } \\
\hline constants & $\leftrightarrow$ & & constants & $\leftrightarrow$ & \\
\hline decrease & $\downarrow$ & & decrease & $\downarrow$ & \\
\hline \multicolumn{2}{|c|}{$\begin{array}{c}\text { Effect of BAY } \\
\text { treatment }\end{array}$} & $\begin{array}{l}\text { length/ } \\
\text { width ratio }\end{array}$ & \multicolumn{2}{|c|}{$\begin{array}{c}\text { Effect of BAY } \\
\text { treatment }\end{array}$} & $\begin{array}{l}\text { length/ } \\
\text { width ratio }\end{array}$ \\
\hline \multicolumn{2}{|c|}{$\begin{array}{c}\text { C ( } \mathrm{n}=12 \text { slices }) \\
\text { vs } \\
\text { C BAY }(\mathrm{n}=14 \text { slices })\end{array}$} & $\mathrm{p}=\stackrel{\leftrightarrow}{0.420}$ & \multicolumn{2}{|c|}{$\begin{array}{c}\mathrm{C}(\mathrm{n}=14 \text { slices }) \\
\text { vs } \\
\text { C BAY }(\mathrm{n}=17 \text { slices })\end{array}$} & $\mathrm{p}=\stackrel{\leftrightarrow}{0.207}$ \\
\hline \multicolumn{2}{|c|}{$\begin{array}{c}\text { CS } 30 \text { (n=6 slices }) \\
\text { vs } \\
\text { CS BAY } 30 \quad(n=14 \\
\text { slices) }\end{array}$} & $\mathrm{p}=\stackrel{\leftrightarrow}{0.131}$ & \multicolumn{2}{|c|}{$\begin{array}{c}\text { CS } 30 \quad(n=11 \text { slices }) \\
\text { vs } \\
\text { CS BAY } 30 \quad(n=12 \\
\text { slices })\end{array}$} & $\mathrm{p}=\stackrel{\leftrightarrow}{0.771}$ \\
\hline \multicolumn{2}{|c|}{$\begin{array}{c}\text { CS } 90(\mathrm{n}=7 \text { slices }) \\
\text { vs } \\
\text { CS BAY } 90 \quad(\mathrm{n}=12 \\
\text { slices) } \\
\end{array}$} & $\mathrm{p}=\stackrel{\leftrightarrow}{0.772}$ & \multicolumn{2}{|c|}{$\begin{array}{c}\text { CS } 90(\mathrm{n}=15 \text { slices }) \\
\text { vs } \\
\text { CS BAY 90 } \quad(n=19 \\
\text { slices })\end{array}$} & $\mathrm{p}=\stackrel{\leftrightarrow}{0.995}$ \\
\hline \multicolumn{3}{|c|}{$\begin{array}{c}\text { Effect of } \\
\text { basal stimulation }\end{array}$} & \multicolumn{3}{|c|}{$\begin{array}{c}\text { Effect of } \\
\text { basal stimulation }\end{array}$} \\
\hline \multicolumn{2}{|c|}{$\begin{array}{c}C(\mathrm{n}=12 \text { slices }) \\
\text { vs } \\
\text { CS } 30 \text { ( } n=6 \text { slices })\end{array}$} & $\mathrm{p}=\stackrel{\leftrightarrow}{0.570}$ & \multicolumn{2}{|c|}{$\begin{array}{c}\mathrm{C}(\mathrm{n}=14 \text { slices }) \\
\text { vs } \\
\text { CS } 30(\mathrm{n}=11 \text { slices })\end{array}$} & $\mathrm{p}=\stackrel{\leftrightarrow}{0.363}$ \\
\hline \multicolumn{2}{|c|}{$\begin{array}{c}\mathrm{C}(\mathrm{n}=12 \text { slices }) \\
\text { vs } \\
\mathrm{CS} 90 \text { ( } \mathrm{n}=7 \text { slices })\end{array}$} & $\mathrm{p}=\overleftrightarrow{0.804}$ & \multicolumn{2}{|c|}{$\begin{array}{c}\mathrm{C}(\mathrm{n}=14 \text { slices }) \\
\text { vs } \\
\text { CS } 90 \text { ( }=15 \text { slices })\end{array}$} & $\mathrm{p}=\stackrel{\leftrightarrow}{0.167}$ \\
\hline
\end{tabular}

Comparison of unstimulated samples at time zero (C) and control slices after 30 min and 90 min of basal stimulation (CS 30, CS 90). The BAY treatment and the basal stimulation did not induce differences between dendritic spine shape in analysed groups (C BAY, CS BAY 30, CS BAY 90).

trains may reflect a decreased $\mathrm{Ca}^{2+}$ entry upon BAY treatment. It is possible that changes in AFP result from BAY-induced drop in extracellular lactate but the precise mechanism remains to be determined. It should be kept in mind that L-lactate may serve also signaling functions that are not exclusively related to energy metabolism. For instance, lactate may act as a signaling molecule enhancing the acid-sensing $\mathrm{Na}^{+}$channel [24] and evoking excitation in locus coeruleus [25]. Although previous studies have demonstrated that application of exogenous L-lactate is sufficient to support LTP in hippocampus formation $[8,16,26]$, the experiments recently performed in our laboratory revealed that L-lactate was able to support LTP in Sch-CA1 pathway only in young but not aged animals (unpublished data). In addition, it is not entirely clear to what extent the impact of BAY concerns pre- or postsynaptic mechanisms. Importantly, longterm BAY treatment did not affect the basal transmission or short term facilitation assessed as PPF (Figure 1B) in young and aged rats, indicating the lack of effect on presynaptic mechanisms under these conditions. However, whereas BAY had no effect on PTP in young animals, in the aged group, PTP was increased in the presence of this compound. This observation suggests that differences in mechanisms of BAY effect on LTP in the two groups may involve some presynaptic mechanisms. Moreover, in both young and aged group, HFS led to a significant reduction of PPF but BAY did not influence this effect. In general, changes in short term plasticity parameters such as PPF or PTP may indicate a presynaptic change (e.g. [27, 28] and in considered Sch-CA1 pathway, a presynaptic component of LTP has been described [29] and therefore a presynaptic BAY action cannot be excluded. On the other hand, attenuation of PPF following LTP induction could also result from altered responsiveness of post-synaptic receptors due to post-synaptic calcium signaling $[30,31]$. It seems thus that differential impact of BAY on late LTP phases in young and old groups appears most consistent with postsynaptic effects while the contribution from presynaptic mechanisms remain to be established.

Intriguingly, basal and HFS stimulation affected the abundance of mRNA for glycogen metabolism enzymes. The mRNA level for all studied enzymes in astrocytes increased in both age groups after BS. In the case of young animals, HFS stimulation did not cause any further increase of mRNA amount for Gys 1 and Pygb. However, application of BAY precluded stable LTP maintenance (Figure 2) and in these conditions expression of Glns, 
Table 4: Effect of BAY on dendritic spine morphology, of young and aged rats, upon HFS.

\begin{tabular}{|c|c|c|c|c|c|}
\hline increase & $\uparrow$ & \multirow{3}{*}{ young } & increase & $\uparrow$ & \multirow{3}{*}{ aged } \\
\hline constants & $\leftrightarrow$ & & constants & $\leftrightarrow$ & \\
\hline decrease & $\downarrow$ & & decrease & $\downarrow$ & \\
\hline \multicolumn{2}{|c|}{$\begin{array}{c}\text { Effect of } \\
\text { stimulation }\end{array}$} & $\begin{array}{l}\text { length/ } \\
\text { width ratio }\end{array}$ & \multicolumn{2}{|c|}{$\underset{\text { stimulation }}{\text { Effect of }}$ HFS } & $\begin{array}{l}\text { length/ } \\
\text { width ratio }\end{array}$ \\
\hline \multicolumn{2}{|c|}{$\begin{array}{c}\text { CS } 30 \text { ( } \mathrm{n}=6 \text { slices }) \\
\text { vs } \\
\text { LTP } 30(\mathrm{n}=7 \text { slices })\end{array}$} & $\mathrm{p}=0.002$ & \multicolumn{2}{|c|}{$\begin{array}{c}\text { CS } 30 \text { ( } \mathrm{n}=11 \text { slices }) \\
\text { vs } \\
\text { LTP } 30 \text { ( } \mathrm{n}=12 \text { slices })\end{array}$} & $\mathrm{p}<0 . \stackrel{\downarrow}{0.0001}$ \\
\hline \multicolumn{2}{|c|}{$\begin{array}{c}\text { CS BAY } 30 \\
\text { slices }) \\
\text { vs } \\
\text { LTP BAY } 30=14 \\
\text { slices })\end{array}(n=13$} & $\mathrm{p}<0 . \stackrel{\downarrow}{ }<001$ & \multicolumn{2}{|c|}{$\begin{array}{rrr}\text { CS } & \begin{array}{r}\text { BAY 30 } 30 \\
\text { slices })\end{array} & (\mathrm{n}=12 \\
& \mathrm{vS} \\
& \\
\text { LTP } & \begin{array}{c}\text { BAY } 30 \\
\text { slices })\end{array} & (\mathrm{n}=24 \\
& \end{array}$} & $\mathrm{p}<0.0001$ \\
\hline \multicolumn{2}{|c|}{$\begin{array}{c}\text { CS } 90 \text { ( } \mathrm{n}=7 \text { slices }) \\
\text { vs } \\
\text { LTP } 90(\mathrm{n}=12 \text { slices })\end{array}$} & $\mathrm{p}<0.0001$ & \multicolumn{2}{|c|}{$\begin{array}{c}\text { CS } 90 \text { ( } \mathrm{n}=15 \text { slices }) \\
\text { vs } \\
\text { LTP } 90 \text { ( } \mathrm{n}=8 \text { slices })\end{array}$} & $\mathrm{p}<0.0001$ \\
\hline \multicolumn{2}{|c|}{$\begin{array}{c}\text { CS BAY 90 } \\
\text { slices) } \\
\text { vs } \\
\text { LTP BAY } 9012 \\
\text { slices) }\end{array}(n=11$} & $\mathrm{p}<0.0001$ & \multicolumn{2}{|c|}{ 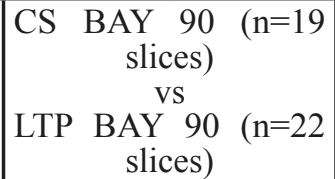 } & $\mathrm{p}<0.0001$ \\
\hline
\end{tabular}

Comparison of basal stimulated control and LTP induced slices at parallel time point of electrophysiological stimulation (after $30 \mathrm{~min}$ and $90 \mathrm{~min}$ - CS 30, LTP 30 and CS 90, LTP 90). Spines of both, young and aged rats changed after HFS stimulation. Morphological analysis indicates the dendritic spine maturation i.e. spines become mushroom shaped, with lower values of length-to-width ratio.

Table 5: The mean density of spines in analysed groups of rats (data from 90 min of stimulation).

\begin{tabular}{|c|c|c|}
\hline slice group & young & aged \\
\hline LTP 90 & $1.13 \pm 0.13 \mu \mathrm{m}^{-1}$ & $1.10 \pm 0.13 \mu \mathrm{m}^{-1}$ \\
\hline LTP BAY 90 & $1.09 \pm 0.18 \mu \mathrm{m}^{-1}$ & $1.10 \pm 0.10 \mu \mathrm{m}^{-1}$ \\
\hline CS 90 & $1.16 \pm 0.20 \mu \mathrm{m}^{-1}$ & $1.01 \pm 0.06 \mu \mathrm{m}^{-1}$ \\
\hline CS BAY 90 & $1.08 \pm 0.16 \mu \mathrm{m}^{-1}$ & $0.98 \pm 0.09 \mu \mathrm{m}^{-1}$ \\
\hline
\end{tabular}

CS, basal stimulated control; LTP, high-frequency stimulated slices; BAY, indicate the inhibitor presence during experiment.

Pygb and Gys 1 in astrocytes (after HFS) was smaller than in controls (Figure 3). This suggests that BS is able to induce gene expression for key metabolic enzymes which appears to be a preparatory step for HFS-induced plasticity phenomena (LTP). In contrast to Gys1 and Pygb the level of mRNA for Glns was markedly higher after LTP induction than upon BS. Presumably, it reflects an upregulation of Glns following a massive influx of glutamate taken up into astrocytes by excitatory aminoacid transporters (EAATs).

The largest changes in gene expression in young animals occurred in astrocytes whereas in neurons either no change was observed (Glns, Pygb) or increase due to basal stimulation or HFS was much smaller than in astrocytes (Figure 3). Most interestingly, in aged animals,
BS and HFS stimulation enhanced the mRNAs levels both in astrocytes and neurons but, in contrast to young rats, this effect was pronounced in neurons. Noteworthy, the Pygb inhibition in slices from aged animals did not disrupt LTP maintenance (as it did in young animals) even if in both groups BAY administration resulted in down regulation of gene expression for Gys1 and Pygb (Figure 3K, 3J and Figure $3 \mathrm{~N}, 3 \mathrm{M})$. The molecular mechanisms underlying this cross-talk between neuronal activity and gene expression remains unclear. Among potential intracellular effectors involved in neuron-astrocyte interplay, adenylate cyclase (Adc) seems to be particularly interesting as it has been shown that glycogen breakdown and lactate release from astrocytes was stimulated by cAMP [32]. Adenylate cyclase is also known to be activated in response to 
elevation of bicarbonate titer during intense neural activity [32]. Since cAMP may stimulate transcription of several metabolic genes [33] thus, increased level of cAMP may be responsible for the observed elevation of the metabolic enzymes after BS and HFS. However, our finding that Pyg inhibition resulted in the attenuation of the expression of the metabolic enzymes suggests that other molecules, which must come from glycogen-derived glucosyl units, are also engaged in regulation of the expression. It cannot be also excluded that the expression of Gys1 and Pyg in astrocytes is activated by unknown molecule released from neurons as a feedback to increased lactate release from astrocytes.

Our morphological studies showed that in aged rats the laminar structure of the hippocampal formation is severely distorted and beta-amyloid plaques were present (Figure 4). These profound morphological alterations raise several possibilities regarding changes in the neuronalastrocytic cross-talk in aged animals. It is well known that astrocytes form several contacts with neuronal cells, especially at synapses and this observation gave rise to a concept of a "tripartite synapse" $[34,35]$. It is possible that such severe morphological changes in aged rats could affect this intimate astrocyte-neuronal interaction. Presumably, precise arrangement of the tripartite synapses is a prerequisite for proper functioning of astrocyteneuron lactate shuttle. Otherwise, lactate which is not properly taken up by neurons may become toxic and in that case, blockade of glycogen phosphorylase with BAY would be beneficial to the neuronal tissue $[36,37]$. In addition, impaired astrocyte-neuronal communication in aged animals is likely to result in a reprograming of gene expression in neurons resulting in a more abundant mRNA expression for key metabolic enzymes considered here which normally (in young animals) are preferentially expressed in astrocytes.

In conclusion, we provide the first evidence that LTP in the hippocampal Sch-CA1 pathway is differentially modulated by astrocytic metabolism in young and aging animals and that the underlying mechanisms involve altered gene expression of key metabolic enzymes, pharmacological sensitivity and spine geometry.

\section{MATERIALS AND METHODS}

\section{Hippocampal slice preparation and electrophysiology}

Experiments were performed on male Wistar rats divided into two age groups: young (P 27 - 35) and aged (P 550 - 700). Animals were anesthetized with isoflurane and decapitated. All procedures were approved by Local Ethical Commission and an effort was made to minimize the number of animals used for experiments. The brain was quickly removed and transverse slices $(350 \mu \mathrm{m}$ thick) were cut with a vibratome (Leica VT1200S, Germany) in ice cold buffer containing (in $\mathrm{mM}$ ): sucrose $75, \mathrm{NaCl} 87$, $\mathrm{KCl} 2.5, \mathrm{NaH}_{2} \mathrm{PO}_{4} 1.25, \mathrm{NaHCO}_{3} 25, \mathrm{CaCl}_{2} 0.5, \mathrm{MgSO}_{4} 7$, and glucose 25 , oxygenated by saturation with carbogen $\left(95 \% \mathrm{O}_{2}, 5 \% \mathrm{CO}_{2}\right), \mathrm{pH} 7.4$. Slices were transferred to a chamber containing the same carbogen saturated solution and kept at $32^{\circ} \mathrm{C}$ for $15 \mathrm{~min}$. Slices were then kept in a holding chamber at room temperature in an oxygenated artificial cerebrospinal fluid (ACSF) containing (in $\mathrm{mM}$ ): $\mathrm{NaCl} 125, \mathrm{NaHCO}_{3} 25, \mathrm{KCl} 3, \mathrm{NaH}_{2} \mathrm{PO}_{4} 1.25, \mathrm{CaCl}_{2} 2.5$, $\mathrm{MgSO}_{4} 1.2$, glucose $10, \mathrm{pH} 7.4$ for at least $1 \mathrm{~h}$. The same ACSF was used in electrophysiological experiments and the preparation was continuously perfused at $9 \mathrm{ml} / \mathrm{min}$. All solutions were continuously bubbled with $95 \% \mathrm{O}_{2}$ and $5 \% \mathrm{CO}_{2}$.

Synaptic transmission in the Schaeffer collateralCA1 (Sch-CA1) pathway was investigated using field potential recordings as described previously [38]. Briefly, Schaffer collateral axons were stimulated with a bipolar electrode (125 um, FHC, USA), and evoked field excitatory postsynaptic potentials (fEPSPs) were recorded in stratum radiatum of CA1. Stimuli were delivered using a stimulator (A360, World Precision Instruments, USA), whereas extracellular recordings were obtained with borosilicate glass micropipettes filled with ACSF, yielding a resistance of 1 - $3 \mathrm{M} \Omega$. The recorded signal was amplified (DAM80, WPI, USA), low-pass filtered at $3 \mathrm{kHz}$, sampled at $10 \mathrm{kHz}$ (Digidata 1440, Molecular Devices, USA). The electrophysiology data were analyzed using pClamp 10.3 (Molecular Devices) and AxoGraphX (developed by John Clements).

Input-output curves (I-O) were obtained based on recordings of field excitatory postsynaptic potentials (fEPSP) evoked by monotonically increasing stimulus. Basal fEPSPs were recorded at stimulation intensity equal to $30-40 \%$ of that giving maximum response. Synaptic long-term potentiation was evoked by highfrequency stimulation (HFS, 4 x $100 \mathrm{~Hz}, 1$ s duration with $10 \mathrm{~s}$ intertrain intervals). Paired-pulse facilitation (PPF) is expressed as the ratio of fEPSP slope (second/ first) from two responses evoked with stimuli applied with an interstimulus interval of $50 \mathrm{~ms}$. The fEPSP slope was calculated as the value of the maximal slope of the descending phase between $20 \%$ and $80 \%$ of the negative peak response.

Depolarizing envelope area was determined as total area under 100 EPSPs within high frequency train calculated from baseline $(0 \mathrm{mV})$. Positive after potential (after positivity, AFP) was measured at peak occurring at 50 - $100 \mathrm{~ms}$ following high-frequency trains. The stimulus intensity was identical to basal stimulation (approx. 30 $40 \%$ max. response).

A group of slices (labeled in the tables and figures as a "BAY") was additionally incubated with $5 \mu \mathrm{M}$ glycogen phosphorylase allosteric inhibitor (BAY U6751 
- shortly BAY; Santa Cruz, USA) which was added to the incubation medium $30 \mathrm{~min}$ before LTP induction $\left(K_{i}=1.6\right.$ $n M$, soluble in $\mathrm{H}_{2} \mathrm{O}$ ).

\section{Tissue preparation}

For in situ hybridization and immunohistochemistry staining, brain slices ( $350 \mu \mathrm{m}$ thick) were immersed in an alcoholic fixative (1 methanol : 3 ethanol $95 \%$ ) for $20 \mathrm{~min}$ at $4{ }^{\circ} \mathrm{C}$ and subsequently kept at $-20^{\circ} \mathrm{C}$. Fixed slices were washed in $99.8 \%$ ethanol $\left(4^{\circ} \mathrm{C}\right)$ and processed through several mixtures of ethanol and increasing concentration of low melting polyester wax (Electron Microscopy Sciences, USA) until slices were embedded in pure wax. Tissue preparations were cut into $4 \mu \mathrm{m}$ thick sections on rotary microtome (Leica RM 2255, Germany) and mounted on glass slides (Superfrost Plus slides, Menzel Glaser, Germany) and kept at $4^{\circ} \mathrm{C}$. Just before specific staining, tissue sections were dewaxed in $96 \%$ ethanol ( 2 x $5 \mathrm{~min}$ ) and $99.8 \%$ ethanol $(10 \mathrm{~min})$ and rehydrated directly with phosphate saline buffer (PBS).

For morphological analysis of dendritic spines (Diolistic method) brain slices were fixed with $4 \%$ paraformaldehyde in PBS $\left(30 \mathrm{~min}, 4^{\circ} \mathrm{C}\right)$ and kept in cold PBS until subsequent use.

\section{Fluorescence in situ hybridization (FISH) and immunofluorescence staining (IF)}

Dewaxed and rehydrated $4 \mu \mathrm{m}$ thick sections were fixed with $4 \%$ PFA / PBS $\left(10 \mathrm{~min}, 4^{\circ} \mathrm{C}\right)$. Then, to localize mRNAs the slides were washed with sodium citrate buffer (SSC: $150 \mathrm{mM} \mathrm{NaCl}, 15 \mathrm{mM}$ sodium citrate; $\mathrm{pH}$ 7.0) and incubated with hybridization mixture contained $1 \mu \mathrm{M}$ specific Cy3-labeled oligonucleotide to mRNA identification, for $3 \mathrm{~h}$ at $45^{\circ} \mathrm{C}(5 \%$ dextran sulfate sodium salt, $50 \%$ formamide, $0.1 \%$ BSA, $0.1 \%$ Ficoll- 400,5 $\mathrm{mM}$ EDTA, $0.1 \%$ polyvinylpyrrolidone, $50 \mu \mathrm{g} / \mathrm{ml}$ yeast tRNA, $200 \mu \mathrm{g} / \mathrm{ml}$ DNA from salmon testes, $750 \mathrm{mM}$ $\mathrm{NaCl}, 75 \mathrm{mM}$ sodium citrate; $\mathrm{pH}$ 7.0). List of 5'-end Cy3labeled oligonucleotides complementary to studied rat mRNA sequences (Table 1) were synthesized by SigmaLife Science. The FISH reaction was stopped by washing with $2 \times \mathrm{SSC}$ buffer $\left(3 \times 10 \mathrm{~min}, 45^{\circ} \mathrm{C}\right)$.

In order to distinguish astrocytes from neurons, the astrocyte (GFAP) and neuron (MAP2) markers were localized using immunofluorescence staining directly after FISH. For this purpose, slices were incubated for 30 min at $37^{\circ} \mathrm{C}$ with primary antibodies diluted in $2 \times \mathrm{SSC}$ buffer (anti-GFAP, 1:500, Sigma-Aldrich, cat nr G3893; anti-MAP-2, 1:250, Sigma, cat. nr M4403,) and washed with $0.05 \%$ Tween-20/2 x SSC ( 1 x 5 min). Subsequently, slices were incubated for $30 \mathrm{~min}$ at $37^{\circ} \mathrm{C}$ with secondary antibodies diluted in $2 \times \mathrm{SSC}$ buffer (AlexaFluor 633 goat anti-mouse, 1:500, Invitrogen), and washed again. Finally, the slices were stained DAPI (Sigma) to visualize the cell nuclei and mounted in Fluoroshield. In control reaction, the oligonucleotide probes were omitted. All solutions were prepared using DEPC-water and with RNase free equipment.

Particular care was taken that sample pairs of unstimulated control (C), basal stimulated control (CS, we used basal stimulation of $0.1 \mathrm{~Hz}$ lasting 90 minutes), HFS stimulated slices (LTP, lasting 90 minutes) as well as LTP induced slices in BAY inhibitor presence (labeled in the tables and figures as a "LTP BAY"), were from the same animal and were processed simultaneously and subsequently imaged with identical acquisition parameters (i.e. camera gain, exposition time). We tested brain slices of 4 animals for each age and treatment group. Average number for analyzed cells in probe was $n=2282$ and $n=$ 1049 for astrocytes and neurons respectively. The relative fluorescence was normalized as a percentage of initial mRNA level defined for unstimulated control samples (C).

\section{Morphological analysis of dendritic spines}

Random dendrite labelling with a gene gun, using $1.6 \mathrm{~mm}$ tungsten particles (BioRad) coated with propelled lipophilic fluorescent dye (DiI; Invitrogen), was performed for the dendritic spine morphological analysis. Images of secondary apical dendrites located at $50 \mu \mathrm{m}-200 \mu \mathrm{m}$ from the cell soma of the CA1 field of hippocampus were analyzed. The purpose of this restriction was to eliminate possible systematic differences in spine morphologies that are due to the location of spines on dendrite with different ranks. Images were acquired under $561 \mathrm{~nm}$ fluorescent illumination using a confocal microscope ( $40 \mathrm{x}$ objective) at a pixel resolution of 1024 x 1024 with a 3.33 zoom, which resulted in a $72 \mathrm{~nm}$ pixel size. Spines were measured and analyzed using semiautomatic, custom written software, Spine Magic [39]. We determined a scale-free parameter, the length-towidth ratio (i.e. the length divided by the width), which reflects the spine shape. We analyzed spine morphology of young ( 1 month) and aged (20-22-months) rats. 3 young and 4 aged rats were analyzed. The hippocampal slices of each rat were divided into ten experimental groups (Table 2). From each of the group, at least 300 spines were analyzed. Additionally, control groups were analyzed: C, C BAY, where hippocampal slices were fixed after brain preparation, without any exogenous stimulation.

\section{Immunohistochemistry (IHC)}

Tissue sections were dewaxed in alcohol $(96 \%, 100$ $\%)$ in $37^{\circ} \mathrm{C}$ and fixed in paraformaldehyde $\left(10 \mathrm{~min}, 4^{\circ} \mathrm{C}\right)$. The IHC reactions were performed in the Autostainer Link 48 (Dako, Glostrup, Denmark) using an EnVision FLEX visualization system (Dako). Endogenous peroxidase was 
blocked by incubating sections in $3 \%$ hydrogen peroxide. For detection beta-amyloid sections were incubated with the primary antibody diluted at $1: 400$ (monoclonal mouse antibody; Convance, cat. no SIG 39320-1000) for $20 \mathrm{~min}$ at room temperature. Secondary goat antibody (EnVision/HRP; Dako) were linked to horseradish peroxidase. The substrate for the reaction was DAB (3,3'- diaminobenzidine tetrachlorohydrate). The slices were counterstained with hematoxylin and coversliped with Mounting Medium (Dako, Denmark). Sections immunostained in the absence of primary antibody were used as negative controls.

\section{Image acquisition}

Wide-field FISH+IF fluorescence was acquired with fluorescence microscope Olympus BX51 combined with Olympus DP72 camera. For each slice pictures were analyzed for: (1) number of nuclei (DAPI at $430 \mathrm{~nm}$ ), (2) relative fluorescence for mRNA concentration $(\mathrm{Cy} 3$ at $540 \mathrm{~nm}$ ) and (3) for brain cell distinction between neurons and astrocytes (GFAP/MAP-Alexa fluor at 633 $\mathrm{nm})$. For IHC image acquisition we used VIS microscope Olympus BX41 combined with Olympus ColorView III camera. For each hippocampi a series of pictures with highest resolution was acquired $(40 \mathrm{x}$ objective at a pixel resolution of $4140 \times 3096$ ).

\section{Image analysis}

The image analysis was performed in the following steps. First, areas of 6 - 8 adjacent visual fields (approximately $200 \times 180 \mu \mathrm{m}$ each) for CA1 regions were analyzed within each thin $(4 \mu \mathrm{m})$ hippocampal section. To build proper statistics, the measurements were repeated for 4 independent hippocampal preparations representing the same type of electrophysiological experiment ( $4 \mathrm{x} \mathrm{C}$, 4 x CS, 4 x LTP, 4 x LTP BAY). Then Cy3 fluorescence values within each group were pooled, providing a final relative fluorescence value to create the histogram curves. Such analysis was performed in hippocampal sections stained against microtubule associated protein 2 (MAP-2), a neuronal marker. In our hands, MAP-2 antibody stained both soma and dendrites of rat hippocampal neurons and therefore it was used as a reference of neuronal structure (Figure 3A-3D). Parallel hippocampal sections were stained additionally against GFAP protein, which enabled us to analyze astrocyte soma with astrocyte tabs (Figure $3 \mathrm{E}-3 \mathrm{H})$. The analysis of mean $\mathrm{Cy} 3$ fluorescence was performed within regions of interest (ROIs) which were exclusively in MAP-2 positive cells (i.e. neurons, Figure 3D) of CA1 stratum pyramidale, within GFAP positive cells (i.e. astrocyte) in the same region and within GFAP positive cells in CA1 stratum radiatum (Figure $3 \mathrm{H}$ ). Image analysis was performed using Olympus $\mathrm{Cell}^{\wedge} \mathrm{F}$ software.
Fluorescence intensity for $\mathrm{Cy} 3$ staining, corresponding to mRNA level in neurons or astrocytes was corrected by subtracting the background fluorescence level.

\section{Statistical analysis}

Data were analyzed using unpaired t-test or ANOVA. Significance in the ANOVA was followed by the Holm-Sidak post-hoc test. The statistical analyses were performed using SigmaStat 3.1 software (Systat Software). Data are expressed as mean \pm SEM with significance levels of $* p \leq 0.05, * * p \leq 0.01, * * * p \leq 0.005$. In electrophysiology results, $n$ refers to the number of individual slices from different animals. In FISH and IF $n$ refers to number of analyzed cells (astrocytes or neurons).

For dendrite spine morphology analysis, the data are expressed as mean $+/$ - standard error of the mean (SEM). Datasets were tested using the two-tailed Student's t-test and if the number of groups were larger than two, one-way ANOVA was used. For statistical analysis of cumulative distribution plot Kolmogorov-Smirnov test were employed. Statistical analyses were performed using Origin 8 software (Origin Lab Corporation).

\section{ACKNOWLEDGMENTS}

We thank Piotr Dzięgiel, Karolina Jabłońska and Agnieszka Barańska from Department of Histology and Embryology, Wroclaw Medical University for technical assistance. We also thank Piotr Mamczur from Department of Animal Molecular Physiology (Wroclaw University, Poland) for designing Cy3 oligonucleotides.

\section{CONFLICTS OF INTEREST}

We declare no financial interests or conflicts of interest.

\section{GRANT SUPPORT}

This work was supported by the Polish Ministry for Science and Higher Education grant No.: N N303 815540.

\section{REFERENCES}

1. Rosenzweig ES, Barnes CA. Impact of aging on hippocampal function: plasticity, network dynamics, and cognition. Prog Neurobiol. 2003; 69:143-179

2. Henley JM, Wilkinson KA. AMPA receptor trafficking and the mechanisms underlying synaptic plasticity and cognitive aging. Dialogues Clin Neurosci. 2013; 15:11-27

3. Bourne JN, Harris KM. Coordination of size and number of excitatory and inhibitory synapses results in a balanced structural plasticity along mature hippocampal CA1 
dendrites during LTP. Hippocampus. 2010; 21:354-373

4. Petralia RS, Mattson MP, Yao PJ. Communication breakdown: the impact of ageing on synapse structure. Ageing Res Rev. 2014; 14:31-42

5. Parpura V, Zorec R. Gliotransmission: Exocytotic release from astrocytes. Brain Res Rev. 2009; 63:83-92

6. Mozrzymas J, Szczesny T, Rakus D. The effect of glycogen phosphorolysis on basal glutaminergic transmission. Biochem Biophys Res Commun. 2011; 404:652-655

7. Gibbs ME, Anderson DG, Hertz L. Inhibition of glycogenolysis in astrocytes interrupts memory consolidation in young chickens. Glia. 2006; 54:214-222

8. Suzuki A, Stern SA, Bozdagi O, et al. Astrocyte-neuron lactate transport is required for long-term memory formation. Cell. 2011; 144:810-823

9. Gibbs ME, O’Dowd BS, Hertz E, Hertz L. Astrocytic energy metabolism consolidates memory in young chicks. Neuroscience. 2006; 141:9-13

10. Kumar A, Thinschmidt JS, Foster TC, King MA. Aging effects on the limits and stability of long-term synaptic potentiation and depression in rat hippocampal area CA1. J Neurophysiol. 2007; 98:594-601

11. Freeman WM, VanGuilder HD, Bennett C, Sonntag WE. Cognitive performance and age-related changes in the hippocampal proteome. Neuroscience. 2009; 159:183-195

12. Fernandes SP, Dringen R, Lawen A, Robinson SR. Neurones express glutamine synthetase when deprived of glutamine or interaction with astrocytes. J Neurochem. 2010; 114:1527-1536

13. Pellerin L, Pellegri G, Bittar PG, et al. Evidence supporting the existence of an activity-dependent astrocyte-neuron lactate shuttle. Dev Neurosci. 1998; 20:291-299

14. Pellerin L, Magistretti PJ. Sweet sixteen for ANLS. J Cereb Blood Flow Metab. 2012; 32:1152-1166

15. Hertz L, Gibbs ME. What learning in day-old chickens can teach a neurochemist: focus on astrocyte metabolism. J Neurochem. 2009; 109 Suppl 1:10-16

16. Newman LA, Korol DL, Gold PE. Lactate produced by glycogenolysis in astrocytes regulates memory processing. PLoS One. 2011; 6:e28427

17. Gu N, Vervaeke K, Hu H, Storm JF. Kv7/KCNQ/M and $\mathrm{HCN} / \mathrm{h}$, but not $\mathrm{KCa} 2 / \mathrm{SK}$ channels, contribute to the somatic medium after-hyperpolarization and excitability control in CA1 hippocampal pyramidal cells. J Physiol. $2005 ; 566: 689-715$

18. Tzingounis AV, Nicoll RA. Contribution of KCNQ2 and KCNQ3 to the medium and slow afterhyperpolarization currents. Proc Natl Acad Sci U S A. 2008; 105:1997419979

19. Villalobos C, Shakkottai VG, Chandy KG, Michelhaugh SK, Andrade R. SKCa channels mediate the medium but not the slow calcium-activated afterhyperpolarization in cortical neurons. J Neurosci. 2004; 24:3537-3542
20. Moyer JR, Jr., Thompson LT, Black JP, Disterhoft JF. Nimodipine increases excitability of rabbit CA1 pyramidal neurons in an age- and concentration-dependent manner. $\mathrm{J}$ Neurophysiol. 1992; 68:2100-2109

21. Disterhoft JF, Oh MM. Learning, aging and intrinsic neuronal plasticity. Trends Neurosci. 2006; 29:587-599

22. Motanis H, Maroun M, Barkai E. Learning-induced bidirectional plasticity of intrinsic neuronal excitability reflects the valence of the outcome. Cereb Cortex. 2012; 24:1075-1087

23. Sah P, Bekkers JM. Apical dendritic location of slow afterhyperpolarization current in hippocampal pyramidal neurons: implications for the integration of long-term potentiation. J Neurosci. 1996; 16:4537-4542

24. Immke DC, McCleskey EW. Lactate enhances the acidsensing $\mathrm{Na}+$ channel on ischemia-sensing neurons. Nat Neurosci. 2001; 4:869-870

25. Tang F, Lane S, Korsak A, et al. Lactate-mediated glianeuronal signalling in the mammalian brain. Nat Commun. 2014; 5:3284

26. Izumi Y, Katsuki H, Zorumski CF. Monocarboxylates (pyruvate and lactate) as alternative energy substrates for the induction of long-term potentiation in rat hippocampal slices. Neurosci Lett. 1997; 232:17-20

27. Wu LG, Saggau P. Adenosine inhibits evoked synaptic transmission primarily by reducing presynaptic calcium influx in area CA1 of hippocampus. Neuron. 1994; 12:11391148

28. Madronal N, Gruart A, Delgado-Garcia JM. Differing presynaptic contributions to LTP and associative learning in behaving mice. Front Behav Neurosci. 2009; 3:7

29. Bayazitov IT, Richardson RJ, Fricke RG, Zakharenko SS. Slow presynaptic and fast postsynaptic components of compound long-term potentiation. J Neurosci. 2007; 27:11510-11521

30. Wang JH, Kelly PT. Postsynaptic injection of CA2+/CaM induces synaptic potentiation requiring CaMKII and PKC activity. Neuron. 1995; 15:443-452

31. Wang JH, Kelly PT. Attenuation of paired-pulse facilitation associated with synaptic potentiation mediated by postsynaptic mechanisms. J Neurophysiol. 1997; 78:27072716

32. Choi HB, Gordon GR, Zhou N, et al. Metabolic communication between astrocytes and neurons via bicarbonate-responsive soluble adenylyl cyclase. Neuron. 2012; 75:1094-1104

33. Waltereit R, Weller M. Signaling from cAMP/PKA to MAPK and synaptic plasticity. Mol Neurobiol. 2003; 27:99-106

34. Halassa MM, Fellin T, Haydon PG. The tripartite synapse: roles for gliotransmission in health and disease. Trends Mol Med. 2007; 13:54-63

35. Haber M, Zhou L, Murai KK. Cooperative astrocyte and dendritic spine dynamics at hippocampal excitatory 
synapses. J Neurosci. 2006; 26:8881-8891

36. Fischer K, Hoffmann P, Voelkl S, et al. Inhibitory effect of tumor cell-derived lactic acid on human T cells. Blood. 2007; 109:3812-3819

37. Costa Leite T, Da Silva D, Guimaraes Coelho R, Zancan $\mathrm{P}$, Sola-Penna M. Lactate favours the dissociation of skeletal muscle 6-phosphofructo-1-kinase tetramers downregulating the enzyme and muscle glycolysis. Biochem J. 2007; 408:123-130

38. Wiera G, Wozniak G, Bajor M, Kaczmarek L, Mozrzymas JW. Maintenance of long-term potentiation in hippocampal mossy fiber-CA3 pathway requires fine-tuned MMP-9 proteolytic activity. Hippocampus. 2013; 23:529-543

39. Ruszczycki B, Szepesi Z, Wilczynski GM, et al. Sampling issues in quantitative analysis of dendritic spines morphology. BMC Bioinformatics. 2012; 13:213 